\title{
Losses and gains in experiential education in a university pharmacy in Brazil: Lessons from a pandemic
}

\author{
S.N. Leite*, M.R.M. Rover, L. Soares, F.C. Matheus \\ Pharmaceutical Sciences Department - University Pharmacy, Federal University of Santa Catarina, Brazil
}

\section{Keywords}

Experiential Education

Brazil

COVID-19

Pharmacy Education

\section{*Corresponding author:}

silvana.nair.leite@ufsc.br
Summary: The abrupt interruption of planned activities at the University Pharmacy (UP) in the Federal University of Santa Catarina forced a rapid change in routine services with the aim of ensuring continued access to medicines. Since March 2020, the UP has not been able to provide dispensing services or consultations inside its facility because of safety concerns around the spread of COVID-19. Internships are now dedicated to management tasks such as preparing documents to advise patients about particular medicines' utilisation. Virtual communication with the patients was established. This allowed students to be put in charge of keeping in touch with special groups of patients by telephone to track health outcomes, COVID-19 symptoms, and other particular needs. The Global Competency Framework and the National Pharmaceutical Education Guidelines were used as guides to develop the study. Three domains were affected by gains and losses: organisation and management competencies, professional/personal competencies, and pharmaceutical care.

\section{Background and Context}

The rapid spread of coronavirus required adaptability from everyone at University Pharmacy (UP) to manage the ongoing needs of patients, such as access to medicines in a context of social distancing. Many health services avoided the presence of working students to reduce the risk of transmission of COVID-19. The activities of pharmacists have changed in response to the drop in face-to-face services and the reduction of the workforce. The safety of students and faculty members is another important consideration (Fuller et al., 2020).

During this period, in which Brazil is implementing regulations in social distancing which has caused the activity of various sectors to be greatly changed, it has become an imperative to reflect on the impact of this scenario on the experiential education in pharmacy. The abrupt interruption of planned activities at UP in the Federal University of Santa Catarina (UFSC) forced a rapid change in routine services. The changes aimed at ensuring continued access to medicines for patients who were dependent on that pharmacy (a unit of the public health system).
The engagement of students (interns in pharmaceutical practices) has been essential for the pharmacy's ability to serve all patients. As a result, experiential learning has needed to undergo changes to comply with new regulations, and these changes are analysed in this report.

\section{Educational Description}

In Brazil UP is a training provider teaching clinical practice to students as it provides students with direct contact to real patients and real cases. Since 2008, UFSC UP has dispensed high-priced medicines, such as for rheumatoid arthritis, multiple sclerosis, Alzheimer's disease, epilepsy, transplant immunosuppression, lupus erythematosus, and others. These medicines are provided free of charge by the Unified Health System. Many of the medications are prescribed to patients considered to be at high risk if they contract COVID-19. The total number of regular registered patients attending UP is around 5,000 per month, as described in the report by Foppa and colleagues (2020).

Since March 2020, UP has not been able to provide dispensing services or consultations inside its facility because of safety 
concerns associated with COVID-19. The UP prioritised ensuring the delivery of medicines to all patients. This called for changes in routines of the workforce, including the routines of intern students. They are now dedicated to management tasks such as remotely assessing prescriptions, separating and packaging medicines, and preparing documents to advise patients about particular medicines' utilisation. Moreover, virtual communication with patients was established, allowing students to be placed in charge of keeping in touch with specific groups of patients (asthma, arthritis, obstructive lung disease, heart diseases) by telephone to track health outcomes, COVID-19 symptoms and other particular needs.

With these measures, UP has been able to ensure continued access to medicines and experiential learning for the pharmacy internship students. In order to assess the impact of the changes made in services in the experiential learning offered, the pre-pandemic and current internship activities were described and analysed in terms of competencies addressed. The Global Competency Framework (FIP, 2017) and the National Pharmaceutical Education Guideline (National Council of Education, 2017) were used as guides to develop the study. The authors analysed the possible learning losses and gains caused by the UP services' changes. Three domains were affected by both losses and gains.

\section{Gains}

Organisation and Management Competencies

Behaviours - the tasks related to management, including participation in the decisions about the changes in the service routines, are new for the students, and involved: identifying and implementing new services (according to local needs); developing knowledge and practice in storing medicines to minimise errors and maximise accuracy; addressing and managing routine management issues; developing the ability to take accurate and timely decisions and make appropriate judgments.

\section{Professional/Personal Competencies}

Behaviours - The most important change was the shift from face-to-face contact with patients to virtual contact. It allowed students to develop new communication skills to communicate with patients; develop cultural behaviours and sensitivity; tailor communications to patients' needs; take responsibility for own actions and patient care; adapt and implement Standing Operating Procedures (SOPs); prioritise work and implement innovative ideas.

\section{Losses}

Pharmaceutical care

Comparing with the pre-pandemic period, fewer opportunities have been available for the development of some behaviours such as using dispensing devices; applying first aid and arranging follow-up care; assessing based on subjective measures.

\section{Outcomes and Recommendations}

The disruption occurred literally overnight, requiring pharmacists to demonstrate significant adaptability and resilience (Fuller et al., 2020) to manage continuing and, in some cases, expanding needs of patients to access their medicines in a context of social distancing. Important outcomes to be highlighted include medication dispensing was maintained for all patients; the dispensation time was reduced; no staff member or student has yet been infected; students developed management skills; and students also learned how to manage patient care remotely. It can be seen that it is possible to maintain student learning through experiential learning.

Some recommendations to be highlighted are adapting procedures in services for dispensing medication is necessary so that work teams and interns have time to focus on remote patient care and medication management issues; avoiding shortages and economic losses; having more robust strategies for remote pharmaceutical services; and consequently developing clinical skills in remote experiential learning is urgent. Through the communication channels, several patients reported that they were enjoying the services being offered during the pandemic. It was observed that the students were more involved with management services and were questioning medication logistics and regulations more. The students were more engaged as a work team and acted with more responsibility.

The UP managers and staff are investing in developing a platform for remote interaction with patients, focused on pharmaceutical care and administrative solutions for the dispensing processes. The experiential learning is essential for knowledge creation, since learning takes place not only in the cognitive domain but also in the affective domain, based on perception and action (Kolb, 2015). The experience of UP shows that giving students the chance to deeply engage with decision-making in crisis scenarios is a great opportunity to develop their professional skills.

\section{References}

FIP. (2017). Pharmacy Education Taskforce: A Global Competency Framework v1. Available at: https://www.fip.org/files/fip/ PharmacyEducation/GbCF v1.pdf

Foppa, A.A., Gomes L.O., Rover, M.R.M., dos Santos, R.I, Farias, M.R., \& Leite, S.N. (2019). Teaching and Learning Pharmacy Services: A Teaching Method for Developing Competencies for Patient-Centered Care Through Experiential Learning in a Real Workplace. Journal of Pharmacy Practice, 1-8, https://doi.org/10.1177/0897190019854573

Fuller, K.A., Heldenbrand, S.D., Smith, M.D., \& Malcom, D.R. (2020). THE COVID-19 PANDEMIC ACROSS THE ACADEMY A Paradigm Shift in US Experiential Pharmacy Education Accelerated by the COVID-19 Pandemic. American Journal of Pharmaceutical Education, 84(6), https://doi.org/10.5688/ajpe8149

Kolb, D.A. (2015). Experiential Learning: Experience as the Source of Learning and Development. 2nd ed. Hoboken, NJ: Pearson Education

National Council of Education. (2017). Brasil Conselho nacional de educação. Câmara de educação superior. Resolução CNE/CES n. 6, de 19 de outubro de 2017. Available at: http://portal.mec.gov.br/ docman/outubro-2017-pdf/74371-rces006-17-pdf/file 